Pacific Journal of Mathematics

A BOUND FOR THE ORDERS OF THE COMPONENTS OF A
SYSTEM OF ALGEBRAIC DIFFERENCE EQUATIONS 


\section{A BOUND FOR THE ORDERS OF THE COMPONENTS OF A SYSTEM OF ALGEBRAIC DIFFERENCE EQUATIONS}

\section{BeRnard GREenspan}

1. The object of this paper is to obtain a bound for the orders of the components of a system of algebraic difference equations, each component of which is of dimension zero. In the analytic case, this roughly amounts to determining the maximum number of arbitrary functions of period unity which each corresponding manifold can possess.

2. We deal with difference polynomials in $n$ indeterminates $y_{1}, \cdots, y_{n}$ having coefficients in an inversive difference field, $\mathscr{F}$, of characteristic zero. Transforms are denoted by means of a second subscript appended to Latin letters having a single subscript. Thus, for example, $A_{3,4}^{(2)} \mathrm{de}-$ notes the fourth transform of $A_{3}^{(2)}$. The symbol $\mathscr{F}\left\{y_{1}, \cdots, y_{n}\right\}$ denotes the ring of difference polynomials in the indeterminates $y_{1}, \cdots, y_{n}$. The perfect difference ideal generated by a system $\Phi$ of difference polynomials is designated $\{\Phi\}$. Unless there is a possibility for confusion, the term "ideal" is used for the longer "reflexive difference ideal". It is well known that every perfect ideal is the intersection of a finite number of prime ideals, none of which contain any other, [4]. As in ordinary or in differential algebra, these prime ideals are termed components of the decomposition of the perfect ideal.

If $\Lambda$ is a prime ideal in $\mathscr{F}\left\{u_{1}, \cdots, u_{q} ; y_{1}, \cdots, y_{p}\right\}$, then the $u_{i}$ are said to constitute a parametric set of indeterminates, or briefly parameters, of $A$ if

(1) $\Lambda$ contains no nonzero difference polynomial in the $u_{i}$ alone;

(2) for each $k, 1 \leqq k \leqq p$, there exists in $A$ a nonzero difference polynomial in $y_{k}$ and $u_{1}, \cdots, u_{q}$.

It is shown in [1, p. 141] that all parametric sets of a given reflexive prime difference ideal $\Lambda$ contain the same number of parameters. This number is known as the dimension of $\Lambda$, and is briefly denoted $\operatorname{dim} 4$. If the prime ideal has no parameters, we say its dimension is zero.

By the order of a prime ideal $\Lambda$ in $\mathscr{F}\left\{y_{1}, \cdots, y_{n}\right\}$, we mean the algebraic dimension of $\Lambda$, that is $\partial^{0} \mathscr{F}\left(\eta_{1}, \cdots, \eta_{n} ; \eta_{11}, \cdots, \eta_{n 1} ; \eta_{12}, \cdots, \eta_{n 2}\right.$; $\cdots) / \mathscr{F}$ or $\partial^{0} \mathscr{F}<\eta_{1}, \cdots, \gamma_{n}>/ \mathscr{F}$, where $\eta_{1}, \cdots, \eta_{n}$ is a generic zero of $\Lambda$.

A system of difference (differential) polynomials in $\mathscr{F}\left\{y_{1}, \cdots, y_{n}\right\}$ is said to be of type $\left(r_{1}, \cdots, r_{n}\right)$ if $r_{1}, \cdots, r_{n}$ are the maximum orders of the transforms (derivatives) of $y_{1}, \cdots, y_{n}$ respectively that appear in the system.

Received September 4, 1958. The author wishes to thank Professor R. M. Cohn for help in preparing this paper. 


\section{Ritt proved the following theorem in [2].}

If $\Phi$ is a system of nonzero differential polynomials in $\mathscr{F}\left\{y_{1}\right.$, $\left.\cdots, y_{n}\right\}$ of type $\left(r_{1}, \cdots, r_{n}\right)$ and $\Sigma$ is a component of $\{\Phi\}$ of dimension zero, then the order of $\Sigma$ does not exceed $r_{1}+\cdots+r_{n}$.

We shall prove the following analogous, but weaker theorem for a system of difference polynomials.

THEOREM. Let $\mathscr{F}$ be an inversive difference field of characteristic zero. If $\Phi$ is a system of nonzero difference polynomials in $\mathscr{F}\left\{y_{1}\right.$, $\left.\cdots, y_{n}\right\}$ of type $\left(r_{1}, \cdots, r_{n}\right)$ and every component of $\{\Phi\}$ is of dimension zero, then the order of each component is at most $r_{1}+\cdots+r_{n}$.

4. Lemma. Let $A_{1}, \cdots, A_{p}$ be a chain in $\mathscr{F}\left[u_{1}, \cdots, u_{q} ; y_{1}, \cdots, y_{p}\right]$, $A_{i}$ being of class $q+i$. Suppose $A_{1}, \cdots, A_{p-1}$ is a characteristic set of a prime ideal. Then there exist nonzero polynomials $G_{1}, \cdots, G_{r}$ with the following properties

(i) For each $j, 1 \leqq j \leqq r$, the set

$$
A_{1}, \cdots, A_{p-1} ; G_{j}
$$

is a characteristic set of a prime ideal.

(ii) There exists a polynomial $G$ in $u_{1}, \cdots, u_{q}$ and a product $I$ of powers of initials of $A_{1}, \cdots, A_{p-1}$ such that $I\left(G A_{p}-G_{1} \cdots G_{r}\right)$ is a linear combination of $A_{1}, \cdots, A_{p-1}$.

(iii) The $G_{j}$ are of positive degree in $y_{p}$ and the sum of these degrees is the degree of $A_{p}$ in $y_{p}$.

Proof. ${ }^{1}$ Let $(\gamma)=\left(\tau_{1}, \cdots, \tau_{q} ; \eta_{1}, \cdots, \eta_{p-1}\right)$ be a generic zero of the prime ideal. Let $C_{1}, \cdots, C_{r}$ be the irreducible factors of $A_{p}\left(\gamma ; y_{p}\right)$ in $\mathscr{F}(\gamma)\left[y_{p}\right]$. We note that when the coefficients of the $C_{j}$ are written in the form $\varphi / \psi$, each $\psi$ may be chosen to be a polynomial in the $\tau_{i}$ only, and each $\varphi$ may be chosen of degree in $y_{i}$ less than that of $A_{i}$ in $y_{i}$, $(1 \leqq i \leqq p-1)$. Now there exist $G_{j} \in \mathscr{F}\left[u_{1}, \cdots, u_{q} ; y_{1}, \cdots, y_{p}\right],(1 \leqq j \leqq r)$, and $B \in \mathscr{F}\left[u_{1}, \cdots, u_{q} ; y_{1}, \cdots, y_{p-1}\right]$ with $B(\gamma) \neq 0, C_{j}=G_{j}\left(\gamma ; y_{p}\right) / B(\gamma)$, $\operatorname{deg}_{y_{p}} G_{j}=\operatorname{deg}_{y_{p}} C_{j}$. In particular, (iii) holds.

Let $G=B^{r}$. Then $G A_{p}-G_{1} \cdots G_{r}$ vanishes when $\left(u_{1}, \cdots, u_{q}\right.$; $\left.y_{1}, \cdots, y_{p-1}\right)$ is replaced by $(\gamma)$. For some $I$ as described in the lemma

$$
I\left(G A_{p}-G_{1} \cdots G_{r}\right) \equiv C, \quad\left[A_{1}, \cdots, A_{p-1}\right],
$$

where $C \in \mathscr{F}\left[u_{1}, \cdots, u_{q} ; y_{1}, \cdots, y_{p}\right], \operatorname{deg}_{y_{p}} C<\operatorname{deg}_{y_{p}} A_{i},(1 \leqq i \leqq p-1)$.

1 We are indebted to the referee for this proof, which is somewhat shorter than ours which consisted of a modification of an old proof of J. F. Ritt's. 
Since $C\left(\gamma ; y_{p}\right)=0$, it follows that $C=0$, so that (ii) holds.

Letting $\zeta_{j}$ be a root of $C_{j}$, we see that $A_{1}, \cdots, A_{p-1}, G_{j}$ form a characteristic set of the prime ideal with generic zero $\left(\gamma ; \zeta_{3}\right)$. This proves (i).

5.. We now prove the theorem. Let $\Sigma$ be a component of $\{\Phi\}$, If we treat the transforms of $y_{1}, \cdots, y_{n}$ as indeterminates in the algebraic sense, then difference polynomials of $\Sigma$ can be thought of as ordinary polynomials. Let $\Sigma_{u_{1}, \ldots, u_{n}}$ denote the set of difference polynomials of $\Sigma$ considered as algebraic polynomials in the ring $\mathscr{F}\left[y_{1}, \cdots, y_{1 u_{1}}\right.$; $\left.\cdots ; y_{n}, \cdots, y_{n u_{n}}\right]$. It is readily seen that $\Sigma_{u_{1}, \ldots, u_{n}}$ is an algebraic ideal, and as $\Sigma$ is prime, it is a prime ideal.

Denote $\Sigma_{r_{1}, \ldots, r_{n}}$ by $\bar{\Sigma}$. Then $\bar{\Sigma} \supseteqq \Phi$. Assume $r_{1} \geqq r_{2} \geqq \cdots \geqq r_{n}$. Let $r_{i}-r_{i+1}=k_{i},(i=1, \cdots, n-1)$, and $m_{i}=\sum_{j=i}^{n-1} k_{j}$. Consider the following array.

$$
\begin{aligned}
& y_{1}, \cdots, y_{1 k_{1}}, \cdots, y_{1, k_{1}+k_{2}}, \cdots, y_{1 m_{1}}, \cdots, y_{1 r_{1}} \\
& y_{2}, \cdots, y_{2 k_{2}}, \quad \cdots, y_{2 m_{2}}, \cdots, y_{2 r_{2}} \\
& y_{3}, \quad \cdots, y_{3 m_{3}}, \cdots, y_{3 r_{3}} \\
& y_{n}, \cdots, y_{n r_{n}} .
\end{aligned}
$$

For the purpose of constructing a characteristic set of $\Sigma$, let the indeterminates be ordered by reading the foregoing array columnwise. Thus, we have the ordering

$$
\begin{array}{r}
y_{1}, \cdots, y_{1 k_{1}}, y_{2}, \cdots, y_{1, k_{1}+k_{2}}, y_{2 k_{2}}, y_{3}, \cdots, \\
y_{1 m_{1}}, y_{2 m_{2}}, y_{3 m_{3}}, \cdots, y_{n}, \cdots, \\
y_{1 r_{1}}, y_{2 r_{2}}, y_{3 r_{3}}, \cdots, y_{n r_{n}} .
\end{array}
$$

Let $\mathfrak{U}$ denote the characteristic set of $\bar{\Sigma}$ which we are going to construct with respect to the ordering (2). Denote the polynomial of $\mathfrak{A}$ which introduces $y_{i j}$ by $A_{i}^{(j)}$. We shall show

$(\alpha 1)$ If $y_{i n}, 0 \leqq h<r_{i}$, is introduced by a polynomial in $\mathfrak{A}$, then $y_{i, h+1}$ is introduced by a polynomial in $\mathfrak{A} ;^{2}$

( $\alpha 2) \quad y_{i r_{i}},(i=1, \cdots, n)$, is introduced by a polynomial of $\mathfrak{A}$.

Let $A_{j}$ denote the $j$ th polynomial of $\mathfrak{A}$. Take $A_{1}$ as irreducible. Assume $h \neq r_{i}$ and $A_{i}^{(h)}=A_{a}$. In the construction of $\mathfrak{A}$, suppose all letters of (2) up to but not including $y_{i, h+1}$ have been considered. Thus, if

$$
A_{1}, \cdots, A_{b}
$$

${ }^{2} A_{i}^{(0)}$ will denote the polynomial of $\mathfrak{A}$ which introduces $y_{i}$. The symbol " $y_{i 0}$ " sometimes will be used to designate $y_{i}$. 
is the beginning of the characteristic set of $\bar{\Sigma}$ so far constructed, then $1 \leqq a \leqq b$. Consider the difference polynomials of $\Sigma$ as ordinary algebraic polynomials and let $\Omega_{j},(j=1, \cdots, b)$, denote the set of all polynomials of $\Sigma$ of class not more than the class of $A_{j}$ relative to the ordering (2). $\Omega_{j}$ will then be a prime algebraic ideal having $A_{1}, \cdots, A_{\text {f }}$ as its characteristic set.

Let $R$ be the algebraic remainder of $A_{a 1}$ with respect to (3). Then there is a relation

$$
R=B A_{a 1}+K_{1} A_{1}+\cdots+K_{b} A_{b},
$$

where $B$ is a product of powers of the algebraic initials of $A_{1}, \cdots, A_{b}$, and $K_{1}, \cdots, K_{b}$ are polynomials. Since $A_{a} \in \Sigma, A_{a 1} \in \Sigma$; and as $h<r_{i}$, $A_{a 1} \in \bar{\Sigma}$. Therefore, $R \in \bar{\Sigma}$. Let $\gamma$ be the highest power of $y_{i, h+1}$ that appears in $A_{a 1}$ and let $\bar{R}$ be the coefficient of $y_{i, h+1}^{\gamma}$ in $R$. Then

$$
\bar{R}=B \bar{A}_{a 1}+\bar{K}_{1} A_{1}+\cdots+\bar{K}_{b} A_{b},
$$

where $\bar{A}_{a 1}$ is the transform of the algebraic initial of $A_{a}$ and $\bar{K}_{1}, \cdots, \bar{K}_{b}$ are the coefficients of $y_{i, h+1}^{\gamma}$ in $K_{1}, \cdots, K_{b}$ respectively. Now as $B$ $\notin \bar{\Sigma}$, we see that $B, A_{a 1} \notin \Sigma$. Thus, as each of $A_{1}, \cdots, A_{b}$ belongs to $\Sigma$, it follows that $\bar{R} \notin \Sigma$, whence a fortiori is not zero. Therefore, $R$ effectively involves $y_{i, h+1}$, and $\bar{R}$ is its algebraic initial.

Now

$$
A_{1}, \cdots, A_{b} ; R
$$

may be a characteristic set of some prime algebraic ideal. If not, then by the lemma of $\S 4$, there is a polynomial $G$ such that

$$
\left.C\left(G R-G_{1} \cdots G_{r}\right) \equiv 0, \quad \quad \mid A_{1}, \cdots, A_{b}\right],
$$

where $C$ is a product of nonnegative integral powers of the initials of $A_{1}, \cdots, A_{b}$ and the $G_{j}$ are nonzero polynomials such that the sum of the degrees in $y_{i ; l+1}$ is the degree of $R$ in $y_{i, l+1}$. Moreover, for each $j$, $1 \leqq j \leqq r$,

$$
A_{1}, \cdots, A_{b} ; G_{j}
$$

is a characteristic set of a prime ideal. Since $A_{1}, \cdots, A_{b}, R$ belong to $\bar{\Sigma}$, while $C$ does not, at least one of $G_{1}, \cdots, G_{r}$ is in $\bar{\Sigma}$, say $G_{1}$.

If (4) is a characteristic set of a prime algebraic ideal, designate this ideal by $\Omega_{b+1}$ and rename $R, A_{b+1}$. If not, let $G_{1}$ be $A_{b+1}$ and $\Omega_{b+1}$ be the prime algebraic ideal of which $A_{1}, \cdots, A_{b}, G_{1}$ is the characteristic set, Thus, a polynomial $A_{b+1}$ in $\bar{\Sigma}$ has been obtained such that

$$
A_{1}, \cdots, A_{b}, A_{b+1}
$$


is a characteristic set of some prime algebraic ideal, $\Omega_{b+1}$ in $\mathscr{F}\left[y_{1}\right.$, $\left.\cdots, y_{i, h+1}\right] .^{3}$ The initial, $\bar{A}_{b+1}$, of $A_{b+1}$ is reduced with respect to $A_{1}, \cdots, A_{b}$ and is lower than $A_{b+1}$, whence is not contained in $\bar{\Sigma}$. Let the set of all polynomials (considered algebraically) of $\Sigma$ of class not exceeding that of $A_{b+1}$ be designated $\Sigma^{\prime}$. Then

$$
\Omega_{b+1} \subseteq \Sigma^{\prime},
$$

since the polynomials (5) are in $\Sigma^{\prime}$, while their initials are not.

In the characteristic set, (5), of $\Omega_{b+1}$, let $A_{b}, A_{b+1}$ respectively introduce the $c$ th and $d$ th letters of the ordering (2). Now, as is well known, the dimension of an ideal equals the number of indeterminates diminished by the length of a characteristic set. Consequently,

$$
\operatorname{dim} \Omega_{b+1}=d-(b+1) .
$$

Since $A_{1}, \cdots, A_{b}$ are polynomials at the beginning of a characteristic set of $\bar{\Sigma}$,

$$
\operatorname{dim} \Sigma^{\prime} \geqq c-b .
$$

Combining (7), (6), and (8), we secure

$$
d-b-1=\operatorname{dim} \Omega_{b+1} \geqq \operatorname{dim} \Sigma^{\prime} \geqq c-b .
$$

If $d-c=1$, then $\operatorname{dim} \Omega_{b+1}=c-b=\operatorname{dim} \Sigma^{\prime}$. Now suppose $d-c>1$. No characteristic set of $\bar{\Sigma}$ contains a polynomial introducing the $d-c-1$ letters between the $c$ th and $d$ th letters of the ordering (2). Therefore, it follows that the length of any characteristic set of $\Sigma^{\prime}$ cannot exceed $b+1$. Consequently, it cannot be that $\operatorname{dim} \Sigma^{\prime}=c-b+j,(j=0,1, \cdots$, $d-c-2)$, for then every characteristic set of $\Sigma^{\prime}$ would have length greater than $b+1$. Hence,

$$
\operatorname{dim} \Sigma^{\prime}=\operatorname{dim} \Omega_{b+1} .
$$

This, together with (6) imply

$$
\Omega_{b+1}=\Sigma^{\prime} .
$$

Thus, (5) is the beginning of a characteristic set of $\bar{\Sigma} . \quad A_{b+1}$ effectively involves $y_{i ; h+1}$ since $R$ does. Therefore, $A_{b+1}$ introduces $y_{i, h+1}$ and may be considered as $A_{i}^{(h+1)}$. Consequently, our assertion $(\alpha 1)$ is established.

We now turn to proving $(\alpha 2)$. By way of contradiction, suppose

$$
A_{1}, \cdots, A_{e}
$$

is a characteristic set of $\bar{\Sigma}$ and that $y_{i, r_{i}}$ is introduced for $f$ values of

3 The dots in " $F\left[y_{1}, \cdots, y_{i, h+1}\right]$ " represent the letters between $y_{1}$ and $y_{i, h+1}$ in the ordering (2). 
$i$, where $f<n$. Let these values be designated $\sigma_{1}, \cdots, \sigma_{f}$ and suppose $\sigma_{1}<\sigma_{2}<\cdots<\sigma_{f}$. Extend the rows of (1) to include all transforms of $y_{1}, \cdots, y_{n}$. Then reading this columnwise, we get an infinite extension of the ordering (2). For convenience we make the following definitions. A polynomial in the ring $\mathscr{F}\left[y_{1}, \cdots, y_{i j}\right]$, where the dots represent the letters between $y_{1}$ and $y_{i j}$ in the extension of the ordering $(2),{ }^{4}$ will be said to be of type $(i, j)$; and if $y_{i}$, effectively appears in the polynomial, it will be said to be of effective type $(i, j)$. Let the set of all polynomials (considered algebraically) of $\Sigma$ of class not exceeding that of $A_{i}$ be denoted $\Sigma_{i},(i=1, \cdots, e)$. We have previously obtained a prime algebraic ideal $\Omega_{i}=\Sigma_{i},(i=1, \cdots, e)$ having $A_{1}, \cdots, A_{i}$ as its characteristic set. However, although the method used for getting $\Omega_{i}$ cannot be continued beyond $i=e$, the process will be modified slightly so that an infinite set of prime algebraic ideals $\Omega_{e+j},(j=1,2,3, \cdots)$ will be determined.

Before proceeding, let us make a few observations. Let $A_{\sigma_{1}}^{\left(r_{\sigma_{1}}\right)}=A_{g}$. Suppose $g \neq 1$. Now if $U_{1} \in \Omega_{j}$, where $j<g, A_{j}$ and $A_{k}$ are respectively of effective types $(u, v)$ and $(u, v+1)$, then $U_{11} \in \Omega_{k}$. This follows at once since $U_{11} \in \Sigma_{k}$. On the other hand, if $U_{1} \notin \Omega_{j}$, where $j<g$, and is of class not exceeding $A_{j}$, then $U_{11} \notin \Omega_{k}$, since otherwise $U_{11}$ would belong to $\Sigma_{k}$ and $U_{1}$ would be in $\Sigma_{j}=\Omega_{j}$.

First we determine $\Omega_{e+1}$; the other $\Omega_{e+j}$ will be obtained inductively. Let $A_{g}$ and $A_{e}$ respectively introduce $y_{u_{1}, v_{1}-1}$ and $y_{u_{2}, v_{2}}$. In the extension of the ordering (2), let $y_{u_{3} v_{3}}$ be the letter that immediately follows $y_{u_{2} v_{2}}$, and $y_{u_{4} v_{4}}$ the one that immediately precedes $y_{u_{1} v_{1}}$. If $R_{g}$ is the algebraic remainder of $A_{g 1}$ with respect to (10), then there is a relation

$$
I_{e} A_{g 1}-R_{g} \equiv 0, \quad\left[A_{1}, \cdots, A_{e}\right],
$$

where $I_{e}$ is a product of nonnegative integral powers of the initials of $A_{1}, \cdots, A_{e}$. Therefore,

$$
I_{e} \bar{A}_{g 1}-\bar{R}_{q} \equiv 0, \quad\left[A_{1}, \cdots, A_{e}\right],
$$

where $\bar{A}_{g 1}$ and $\bar{R}_{g}$ denote the coefficients in $A_{g 1}$ and $R_{g}$ respectively of the highest power of $y_{u_{1} v_{1}}$ in $A_{\theta_{1}}$.

Let $\Omega_{e}^{\prime}$ be the prime ideal in $\mathscr{F}\left[y_{1}, \cdots, y_{u_{1} v_{1}}\right]$ generated by $\Omega_{e}$. The polynomials of $\Omega_{e}^{\prime}$ are those polynomials in $y_{u_{3} v_{3}}, \cdots, y_{u_{1} v_{1}}$ having coefficients in $\Omega_{e}$. It may, of course, happen that $u_{3}, v_{3}$ are respectively equal to $u_{1}, v_{1}$, in which case " $y_{u_{3} v_{3}}, \cdots, y_{u_{1} v_{1}}$ " is to be regarded as simply " $y_{u_{1} v_{1}}$ ". At any rate, we have

$$
I_{e} \bar{A}_{g_{1}}-\bar{R}_{g} \in \Omega_{e}^{\prime} .
$$

4 Here and elsewhere, where no confusion can result, the dots represent the letters of the extension of the ordering (2) between the given letters. 
Now, as $I_{e}$ is free of $y_{u_{3} v_{3}}, \cdots, y_{u_{1} v_{\mathrm{t}}}$ and $\notin \Omega_{e}$, it follows that $I_{e} \notin \Omega_{e}^{\prime}$. We claim that $\overline{A_{g 1}} \notin \Omega_{e}^{\prime}$. To prove this, we write $\overline{A_{g 1}}$ as a polynomial in the letters $y_{u_{3} v_{3}}, \cdots, y_{u_{1} v_{1}}$. Its coefficients are of type $\left(u_{2}, v_{2}\right)$. Regarding $\bar{A}_{g}$ as a polynomial in $y_{u_{3}, v_{3}-1}, \cdots, y_{u_{1}, v_{1}-1}$, its coefficients are of type $\left(u_{2}, v_{2}-1\right)$ unless $v_{2}=0$. If $v_{2}=0$, then the coefficients are of type $\left(u_{5}, v_{5}\right)$, where in the array (1), $y_{u_{5} v_{5}}$ is the last letter which appears in the column headed by $y_{1, r_{1}-1}$. In any case, since these coefficients are reduced with respect to $A_{1}, \cdots, A_{g-1}$, they do not belong to $\Omega_{g-1}$. Therefore, their transforms are not in $\Omega_{e}$. (If $g=1$, we still see that the transforms of the coefficients of $\overline{A_{g}}$ are not in $\Omega_{e}$. For, if they were in $\Omega_{e}$, then they would belong to $\Sigma$, whence $\overline{A_{1}}$ would belong to $\Sigma$, a contradiction.) Consequently, $\bar{A}_{g 1} \notin \Omega_{e}^{\prime}$, as was asserted. It now follows from (12) that $\bar{R}_{g} \notin \Omega_{e}^{\prime}$. This means $\bar{R}_{g} \neq 0$, and so that $R_{g}$ effectively involves $y_{u_{1} v_{1}}$, that is $y_{\sigma_{1}, r \sigma_{1}+1}$. Hence, $\bar{R}_{g}$ is the algebraic initial of $R_{g}$. From (11) we see $R_{g} \in \Sigma$.

If

$$
A_{1}, \cdots, A_{e} ; R_{g}
$$

is a characteristic set of a prime algebraic ideal, we denote this ideal by $\Omega_{e+1}$ and $R_{g}$ by $A_{e+1}$. If (13) is not a characteristic set of any prime algebraic ideal, then by the lemma of $\S 4$, there is a polynomial $H$ such that

$$
J_{e}\left(H R_{g}-H_{1} \cdots H_{q}\right) \equiv 0, \quad\left[A_{1}, \cdots, A_{e}\right],
$$

where $J_{e}$ is a product of powers of the initials of $A_{1}, \cdots, A_{e}$ and the $H_{\mathrm{J}}$ are polynomials of positive degree in $y_{u_{1} v_{1}}$ such that the sum of these degrees is the degree of $R_{g}$ in $y_{u_{1} v_{1}}$. Moreover, for each $j, 1 \leqq j \leqq q$,

$$
A_{1}, \cdots, A_{e} ; H_{j}
$$

is a characteristic set of a prime ideal. From (14) it is seen that some $H_{j}$, say $H_{1}$, belongs to $\Sigma$. Let $H_{1}$ be $A_{e+1}$ and $\Omega_{e+1}$ be the prime algebraic ideal of which $A_{1}, \cdots, A_{e}, H_{1}$ is the characteristic set. Thus, a polynomial $A_{e+1}$ in $\Sigma$ has been obtained such that

$$
A_{1}, \cdots, A_{e+1}
$$

is a characteristic set of some prime algebraic ideal $\Omega_{e+1}$ in $\mathscr{F}\left[y_{1}\right.$, $\left.\cdots, y_{u_{1} v_{1}}\right]$.

Now let us assume as inductive hypotheses:

( $\beta 1)$ If $U_{1} \in \Omega_{h}, h=g-2+j$, and $A_{h}$ and $A_{k}$ are respectively of effective types $(u, v)$ and $(u, v+1)$, then $U_{11} \in \Omega_{k}$.

( $\beta 2)$ If $U_{1} \notin \Omega_{h}, h=g-2+j, U_{1}$ is of type $(u, v), A_{h}$ and $A_{k}$ are of effective type $(u, v)$ and $(u, v+1)$, respectively, then $U_{11} \notin \Omega_{k}$. 
( $\beta 3) \Omega_{e+1}, \cdots, \Omega_{e+j}$ have been constructed by a process similar to the one described on the preceding pages. That is, if

$$
A_{1}, \cdots, A_{e+j-1}
$$

is a characteristic set of $\Omega_{e+j-1}$, the characteristic set of $\Omega_{e+j}$ will be

$$
A_{1}, \cdots, A_{e+j-1}, A_{e+j},
$$

where $A_{e+j}$ is either the algebraic remainder, $R_{g+j-1}$, of $A_{g+j-1,1}$ with respect to (16) or else is one of the $F_{i}$ obtained from a factorization equation of the type

$$
J_{e+j-1}\left(F R_{g+j-1}-F_{1} \cdots F_{r}\right) \equiv 0, \quad\left[A_{1}, \cdots, A_{e+j-1}\right],
$$

where $J_{e+j-1}$ is a product of nonnegative integral powers of the initials of $A_{1}, \cdots, A_{e+j-1}$ and the $F_{i}$ and $F$ are polynomials having properties analogous to those of the $G_{i}$ and $G$, respectively, of the lemma of $\S 4$.

Our hypotheses have been shown to hold when $j=1$ if $g>1$; and, in fact, it has been proven that $(\beta 3)$ is true even if $g=1$. We now verify $(\beta 1)$ and $(\beta 2)$ for $h=1, g=1$; that is, for $j=2, g=1$. Thus, we must prove:

( $\mathscr{1} 1)$ If $g=1$ and $U_{i} \in \Omega_{1}$, then $U_{11} \in \Omega_{e+1}$.

$(\varphi 2)$ If $g=1$ and $U_{1} \notin \Omega_{1}$, where $U_{1}$ is of type $\left(\sigma_{1}, r_{\sigma_{1}}\right)$, then $U_{11} \notin \Omega_{c+1}$. If $U_{1} \in \Omega_{1}$, then for a suitable power $N_{1}$, of the initial $\overline{A_{1}}$ of $A_{1}$, we have

$$
N_{1} U_{1} \equiv 0,
$$

Consequently,

$$
N_{11} U_{11} \equiv 0,
$$

If $N_{11} \in \Omega_{e+1}$, it would then follow that $N_{1} \in \Sigma$, which is false. Therefore, $N_{11} \notin \Omega_{e+1}$. Now either $A_{e+1}$ equals the algebraic remainder, $R_{1}$, of $A_{11}$ with respect to (10), or else $A_{e+1}$ is an $H_{i}$ resulting from a factorization equation of the type (14). In either case, we see that $R_{1} \in \Omega_{c+1}$, whence, from (11), $A_{11} \in \Omega_{e+1}$. Thus, $U_{11} \in \Omega_{e+1}$ and $(\varphi 1)$ is proven.

On the other hand, if $U_{1}$ is of type $\left(\sigma_{1}, r_{\sigma_{1}}\right)$ and $\notin \Omega_{1}$, then $U_{1} \notin \Sigma$, whence $U_{11} \notin \Sigma$. Therefore, $U_{11} \notin \Omega_{e+1}$ and $(\varphi 2)$ is proven.

We are now ready for our induction. We shall prove $(\gamma 1),(\gamma 2),(\gamma 3)$, where these respectively are like $(\beta 1),(\beta 2),(\beta 3)$ with $j+1$ replacing $j$,

Let $U_{1} \in \Omega_{h+1}$ and $A_{h+1}$ be of effective type $(u, v)$. Since $h=g-2+j$ and $e=g-1+f$, it follows that $h+1+f=e+j$. Therefore, by hypothesis $(\beta 3), \Omega_{h+1+f}$ and $A_{h+1+f}$ have been determined. Obviously, $A_{h+1+f}$ is of effective type $(u, v+1)$. Now for a suitable product of powers, $M_{1}$, of the initials of $A_{1}, \cdots, A_{h+1}$, we have 


$$
M_{1} U_{1} \equiv 0, \quad\left[A_{1}, \cdots, A_{l+1}\right] .
$$

Consequently,

$$
M_{11} U_{11} \equiv 0, \quad\left[A_{11}, \cdots, A_{l+1,1}\right] .
$$

By inductive hypothesis ( $\beta 1), A_{11}, \cdots, A_{l 1} \in \Omega_{h+f} \subset \Omega_{h+1+f}$. Let $\Omega_{h+f}^{\prime}$ be the prime algebraic ideal in $\mathscr{F}\left[y_{1}, \cdots, y_{u, v+1}\right]$ generated by $\Omega_{h+f}$. We know that $A_{h}$ introduces some indeterminate, say the letter immediately preceding $y_{u v}^{-}$in the extension of the ordering (2). Also, we know that $A_{l+1}$ introduces $y_{u v}$. We assert that $M_{11} \notin \Omega_{h+1+f}$. To prove this, suppose otherwise. Then $M_{11}$ has zero remainder with respect to $A_{1}, \cdots, A_{l+1+f}$, and since $M_{11}$ is free of $y_{u, v+1}$, it has in fact zero remainder with respect to $A_{1}, \cdots, A_{h+f}$. Thus, $M_{11} \in \Omega_{h+f}^{\prime}$. Hence, if we consider $M_{11}$ as a polynomial in $y_{\bar{u}, v+1}, \cdots, y_{u, v+1}$, its coefficients belong to $\Omega_{l_{t+f}}$. By the induction hypothesis $(\beta 2)$, therefore, the coefficients of $M_{1}$, considered as a polynomial in $y_{\bar{u},}, \cdots, y_{u v}$, are contained in $\Omega_{h} \subset \Omega_{h+1}$. But then $M_{1} \in \Omega_{h+1}$, a contradiction. Hence, our assertion that $M_{11} \notin \Omega_{h+1+f}$ is proved.

If we show that $A_{h+1,1} \in \Omega_{h+1+f}$, then by (18) we shall have $U_{11} \in \Omega_{h+1+f}$ and so $(\gamma 1)$. Now $A_{h+1+f}$ either equals the algebraic remainder, $R_{h+1}$, of $A_{h+1.1}$ with respect to $A_{1}, \cdots, A_{h+f}$ or else is some polynomial $F_{i}$ resulting from a factorization equation of the type (17). That is, we have either

$$
I_{h+f} A_{h+1,1}-A_{h+1+f} \equiv 0, \quad\left[A_{1}, \cdots, A_{h+f}\right]
$$

or taking $A_{h+1+f}$ to be $F_{1}$,

$$
J_{h+f}\left(F R_{h+1}-A_{h+1+f} \cdot F_{2} \cdots F_{s}\right) \equiv 0, \quad\left[A_{1}, \cdots, A_{h+f}\right],
$$

where $I_{l+f}$ and $J_{+f}$ are each products of powers of the initials of $A_{1}, \cdots, A_{\text {ix }+f}$ and $F$ is a polynomial having properties analogous to $G$ of the lemma of $\S 4$.

If (19) is the case, it is immediate that $A_{h+1,1} \in \Omega_{h+1+\jmath}$. On the other hand, if we have (20), then $R_{t+1} \in \Omega_{h+1+f}$, in which case once again we have $A_{h+1,1} \in \Omega_{h+1+f}$. The proof of $(\gamma 1)$ is therefore complete.

We turn now to $(\gamma 2)$. Let $U_{1} \notin \Omega_{h+1}, U_{1}$ be of type $(u, v)$, and $A_{h+1}$ be of effective type $(u, v)$. Then $A_{h+1+f}$ is of effective type $(u, v+1)$. Since every component of the ideal $\left[U_{1}, \Omega_{l+1}\right]$ is of lower dimension than $\operatorname{dim} \Omega_{h+1}$, a polynomial, $V_{1}$, in the parameters of $\Omega_{h+1}$ can be found such that

$$
V_{1}=W_{1} U_{1}+X_{1}
$$

where $X_{1} \in \Omega_{h+1}$ and $W_{1}$ is of type $(u, v)$. From (21) we secure

$$
V_{11}=W_{11} U_{11}+X_{11} \text {. }
$$


Since we just proved $(\gamma 1)$, we see that $X_{11} \in \Omega_{h+1+f}$. If we prove that $V_{11} \notin \Omega_{h+1+f}$, we would have $W_{11} U_{11} \notin \Omega_{h+1+f}$. Then $U_{11} \notin \Omega_{h+1+f}$ and $(\gamma 2)$ would immediately follow.

By way of contradiction, suppose $V_{11} \in \Omega_{h+1+f}$. Then $V_{11}$ has zero remainder with respect to $A_{1}, \cdots, A_{h+1+f}$. Let $A_{\pi_{1}}, \cdots, A_{\pi_{f}}$ be those $A$ 's of the characteristic set, (10), of $\bar{\Sigma}$ which respectively introduce the least transforms of $y_{\sigma_{1}}, \cdots, y_{\sigma_{f}}$. Suppose $A_{{ }_{i}}=A_{\sigma_{i}}^{\left(t_{i}\right)},(i=1, \cdots, f)$. Note that it is not necessarily the case that $\pi_{1}<\pi_{2}<\cdots<\pi_{f}$. Let $\lambda_{1}, \cdots, \lambda_{f}$ be $\pi_{1}, \cdots, \pi_{f}$ arranged in order of increasing magnitude. Then $\lambda_{1}=1$. Now the class of $V_{11}$ in each of the letters $y_{\sigma_{1}}, \cdots, y_{\sigma_{f}}$ does not exceed the class of $A_{\pi_{1}}, \cdots, A_{\pi_{f}}$ in each of these letters respectively. Thus, $V_{11}$ has zero remainder with respect to $A_{1}, \cdots, A_{\lambda_{f}}$. Suppose no transform of $y_{i},\left(i=\rho_{1}, \cdots, \rho_{n-f}\right)$, is introduced by any of the polynomials of (10). Write $V_{11}$ as a polynomial in the $y_{i j}$ following $y_{\sigma_{f} t_{f}}$ in the extension of the ordering (2). These $y_{i j}$ will all be transforms of $y_{\rho_{1}}, \cdots, y_{\rho_{n-f}}$, hence parameters of $\Omega_{h+1+f}$. Therefore, the coefficients will have zero remainder with respect to $A_{1}, \cdots, A_{\lambda_{f}}$, and so belong to $\bar{\Sigma}$. But the inverse transforms are of order less than $t_{i}$ in $y_{\sigma_{i}}, \quad(i=$ $1, \cdots, f)$. As $\bar{\Sigma}$ contains no polynomials of this sort, a contradiction has been obtained. Consequently, $V_{11} \notin \Omega_{h+1+f}$. This proves $(\gamma 2)$.

To establish $(\gamma 3)$, that is to construct $\Omega_{e+j+1}$, one need only to proceed in a manner analogous to the way in which $\Omega_{e+1}$ was determined, except for the specification " $H_{1} \in \Sigma$ ".

Thus, we have demonstrated for all $i$ :

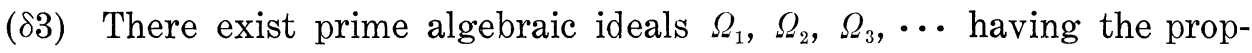
erties

(i) $\Omega_{1} \subset \Omega_{2} \subset \Omega_{3} \subset \cdots$;

(ii) a characteristic set of $\Omega_{i}$ is $A_{1}, \cdots, A_{i}$;

(iii) $\Omega_{j}=\Sigma_{j},(j=1, \cdots, e)$;

(iv) if $\Omega_{g-1+i}$ is an ideal in $\mathscr{F}\left[y_{1}, \cdots, y_{u v}\right]$, then $\Omega_{g-1+i+f}$ is an ideal in $\mathscr{F}\left[y_{1}, \cdots, y_{u v+1}\right]$.

(81) If $U_{1} \in \Omega_{i}$ and $A_{i}$ and $A_{k}$ are respectively of effective types $(u, v)$ and $(u, v+1)$, then $U_{11} \in \Omega_{k}$.

(82) If $U_{1} \notin \Omega_{i}, U_{1}$ is of type $(u, v), A_{i}$ and $A_{k}$ are of effiective types $(u, v)$ and $(u, v+1)$ respectively, then $U_{11} \notin \Omega_{k}$.

Let $\Omega$ be the union of the $\Omega_{i}$ of $(\delta 3) . \quad \Omega$ is obviously a prime algebraic ideal, and indeed, as we shall see, a reflexive prime difference ideal. If $U_{1} \in \Omega$, then there is some $i$ such that $U_{1} \in \Omega_{i}$, whence by ( $\left.\delta 1\right)$, for a suitable $k, U_{11} \in \Omega_{k} \subset \Omega$. Conversely, now suppose $U_{11} \in \Omega$. Then there are positive integers $k, i, u, v$ such that $A_{i}$ and $A_{k}$ are of effective types $(u, v)$ and $(u, v+1)$ respectively, and $U_{11} \in \Omega_{k}$. Therefore, by ( $\delta 2), U_{1} \in \Omega_{i} \subset \Omega$. This proves our assertion that $\Omega$ is reflexive. 
Since the indeterminates $y_{\rho_{1}}, \cdots, y_{\rho_{n-\gamma}}$ are the parameters of $\Omega$, it follows that $\operatorname{dim} \Omega=n-f$. Now $\Omega$ is a divisor of a component of $\{\Phi\}$, say $A$, Therefore, $\operatorname{dim} \Lambda \geqq \operatorname{dim} \Omega=n-f \neq 0$. Hence we have a contradiction of the hypothesis of the theorem that every component of $\{\Phi\}$ is of dimension zero.

Our assertion, $(\alpha 2)$, thus has been established.

Let

$$
A_{1}, \cdots, A_{t}
$$

be a characteristic set of $\bar{\Sigma}$. Before proceeding, several consequences of $(\alpha 1)$ and $(\alpha 2)$ should be noted.

$(\varepsilon 1)$. When we reach the point in the construction of (22) where transforms of each of the letters $y_{1}, \cdots, y_{n}$ have been introduced, all succeeding polynomials of (22) introduce $y_{1}^{\prime} s, \cdots, y_{n}^{\prime} s$ in order, no further transforms from then on being omitted.

$(\varepsilon 2) . \quad y_{1 r_{1}}, \cdots, y_{n r_{n}}$ respectively are introduced by the last $n$ polynomials in $(22)$.

(ع3). In forming (22), certain letters are not introduced by any polynomial of (22). The indeterminates represented by these letters constitute a parametric set of $\bar{\Sigma}$.

If we continue the construction which yielded (22), new polynomials, $A_{i}^{(j)}{ }^{5}$ can be formed such that for any positive integer $m$

$$
A_{1}, \cdots, A_{t} ; A_{1}^{\left(r_{1}+1\right)}, \cdots, A_{n}^{\left(r_{n}+1\right)} ; \cdots ; A_{1}^{\left(r_{1}+m\right)}, \cdots, A_{n}^{\left(r_{n}+m\right)}
$$

is a characteristic set of the prime algebraic ideal $\Sigma r_{1+m, \cdots, r_{n}+m}$ consisting of all polynomials of $\Sigma$ of type $\left(r_{n}, r_{n}+m\right)$ with respect to the extension of the ordering (2). Let this ideal be denoted $\Sigma^{(m)}$. By $(\varepsilon 2),(\varepsilon 3),(\varepsilon 1)$, it follows that the maximum number of parameters in $\Sigma^{(m)}$ for any nonnegative integer $m$ is $r_{1}+\cdots+r_{n}$. Consequently,

$$
\operatorname{dim} \Sigma^{(m)} \leqq r_{1}+\cdots+r_{n} \text {. }
$$

We prove by way of contradiction that the order of $\Sigma$ is at most $r_{1}+\cdots+r_{n}$. Suppose the order of $\Sigma$ is more than $r_{1}+\cdots+r_{n}$. Then for all sufficiently large $a_{1}, \cdots, a_{n}$, the dimension of $\Sigma_{a_{1}, \cdots, a_{n}}$ is greater than $r_{1}+\cdots+r_{n}$, since by definition the order of $\Sigma$ is the algebraic dimension of $\Sigma$. However, this is a contradiction of (23). Hence, the theorem.

6. The bound

$$
r_{1}+\cdots+r_{n}
$$

${ }^{5}$ We are extending the meaning of $A_{i}^{(j)}$, which previously was defined as a polynomial of $\mathfrak{u}$, that is of $(22)$. 
which was obtained in the previous section will be denoted $\mathscr{R}$ and called the Ritt bound. Let

$$
A_{1}^{\left(s_{1}\right)}, \cdots, A_{n}^{\left(s_{n}\right)}
$$

be those polynomials of the characteristic set, $\mathfrak{U}$, of $\bar{\Sigma}$ which respectively introduce the least transforms of $y_{1}, \cdots, y_{n}$. Then $s_{i} \leqq r_{i},(i=$ $1, \cdots, n)$. Then by $(\varepsilon 3)$ it is clear that the order of $\Sigma$ will be given by

$$
\mathscr{S}=s_{1}+\cdots+s_{n} .
$$

In the case of differential equations, Jacobi investigated the problem of determining the number of arbitrary constants in the solution of a system of $n$ equations in the variable $x$ and $n$ dependent variables $y_{1}, \cdots, y_{n}$. If these equations are denoted

$$
B_{i}=0, \quad(i=1, \cdots, n),
$$

and $\alpha_{i j}$ stands for the greatest order of the derivatives of $y_{i}$ in $B_{j}$, then Jacobi asserted, [5] that the number of arbitrary constants in the solution of (26) is no greater than

$$
\max \left(\alpha_{1 j_{1}}+\cdots+\alpha_{n j_{n}}\right)
$$

where $j_{1}, \cdots, j_{n}$ is a permutation of $1, \cdots, n$. However, Jacobi's work was largely heuristic and lacked logical rigor.

Ritt in [2, p. 136] has shown that in the case of two algebraic differential equations in two unknowns, Jacobi is essentially correct. That is, Ritt proved:

If $\Sigma$, of dimension zero, is a component of the system $B_{1}, B_{2}$, then the order of $\Sigma$ is at most $\max \left(\alpha_{11}+\alpha_{22}, \alpha_{12}+\alpha_{21}\right)$.

We shall be interested, in the case of $n$ difference equations in $n$ indeterminates, in obtaining an improvement on the Ritt Bound, and in seeing how it compares with the Jacobi number, (27), where that number now applies to difference polynomials. The number, (27), will be denoted $\mathscr{J}$.

7. Let $F_{1}, \cdots, F_{n}$ be a system of $n$ nonzero difference polynomials of type $\left(r_{1}, \cdots, r_{n}\right)$ in the $n$ indeterminates $y_{1}, \cdots, y_{n}$, where every component of $\left\{F_{1}, \cdots, F_{n}\right\}$ is of dimension zero. Suppose among the $F_{i}$, there is at least one, say $F_{k}$, which does not effectively involve any $y_{j r}$, for $j=1, \cdots, n$. If $F_{k}$ is of effective type $(\theta, \mathcal{D})$, then the characteristic set of $\left\{F_{1}, \cdots, F_{n}\right\}$ certainly must contain a polynomial $A$ of the ring $\mathscr{F}\left[y_{1}, \cdots, y_{\theta \varphi}\right]$. Suppose $A$ is of effective type $(\sigma, \tau)$. By $(\varepsilon 3)$ and $(\alpha 1)$, we are sure, therefore, that $y_{\sigma \tau}, \cdots, y_{\sigma r_{\sigma}}$ are not parameters of $\bar{\Sigma}$, that is of $\Sigma_{r_{1}, \ldots, r_{n}}$. Since $\tau<r_{\sigma}$, we have an improvement on the Ritt bound; $r_{\sigma}$ in (24) is to be replaced by $\tau$. However, we have no simple 
way of determining $\sigma$ and $\tau$. But since $r_{\sigma}-\tau \geqq r_{\theta}-\rho>0$, if we replace $r_{\theta}$ in (24) by $\varphi$, we shall still have a bound which is an improvement on $\mathscr{R}$. As $\theta$ and $\varphi$ are given, the new bound is easily found. Should it happen that several of the $F_{i}$ are devoid of the $y_{j_{r_{j}}}$, then possibly (although not necessarily) we may get a further refinement.

If a transform ${ }^{6}$ of $y_{j}$ appears in $F_{i}$, let $\alpha_{i j}$ stand for the greatest order of the transforms of $y_{j}$ in $F_{i},(i, j=1, \cdots, n)$. For a fixed $i$, consider the set of numbers

$$
r_{k}-\alpha_{i k}, \quad(k=1, \cdots, n) .
$$

(If some $\alpha_{i k}$ are undefined, then (28) will consist of fewer than $n$ numbers). Let $\mathfrak{S}_{i}$ be the set of values of $k$ among $1, \cdots, n$ which will yield the minimum of the numbers (28). If $b_{i}$ denotes the greatest member of $\mathfrak{S}_{i}$, then it will follow that $F_{i}$ is of effective type $\left(b_{i}, \alpha_{i b_{i}}\right)$. Hence, if we replace $r_{b_{i}}$ in (24) by $\alpha_{i b_{i}}$, the result will be an improvement on $\mathscr{R}$ if $F_{i}$ does not effectively involve any $y_{i r_{j}}$.

Let $w=\max \left(r_{b_{i}}-\alpha_{i b_{i}}\right), \quad(i=1, \cdots, n)$, and $\mathscr{C}^{\prime}-w$. Then $\mathscr{G} \leqq \mathscr{R}$, and we have the following

THEOREM. Let $\mathscr{F}$ be an inversive difference field of characteristic zero. If $F_{1}, \cdots, F_{n}$ is a system of $n$ nonzero difference polynomials in $\mathscr{F}\left\{y_{1}, \cdots, y_{n}\right\}$ of type $\left(r_{1}, \cdots, r_{n}\right)$ and every component of $\left\{F_{1}, \cdots, F_{n}\right\}$ is of dimension zero, then the order of each component is at most $\mathscr{S}^{\text {. }}$

8. Although $\mathscr{C}$ is an improvement on $\mathscr{K}$, still in many situations it is larger than $\mathscr{J}$, and of course, under no circumstances ${ }^{7}$ is it less than $\mathscr{J}$. However, we shall show in the case of two nonzero difference polynomials $F_{1}, F_{2}$ in $y_{1}, y_{2}$, that $y=$, whence in such a situation Jacobi's number is a bound. ${ }^{8}$

To prove that $\mathscr{Y}=\mathscr{G}$ in the case of two difference polynomials $F_{1}, F_{2}$ in $y_{1}, y_{2}$, first note that we may assume without loss of generality that $\alpha_{11}=\max \left(\alpha_{11}, \alpha_{12}, \alpha_{21}, \alpha_{22}\right)$. Then $r_{1}=\alpha_{11}$ and $r_{2}=\max \left(\alpha_{12} \alpha_{22}\right)$. It is easily seen that $\mathscr{f}<\mathscr{R}$ if and only if

$$
\alpha_{11}>\alpha_{21} \text { and } \alpha_{12}>\alpha_{22} \text {. }
$$

Now, since $\mathscr{J} \leqq \mathscr{C} \leqq \mathscr{R}$, it follows that if (29) is not satisfied that $\mathscr{F}=\mathscr{G}=\mathscr{R}$. Therefore, suppose the condition (29) holds. In such

${ }^{6}$ Recall $y_{j}$ itself is considered as the zero-th transform of $y_{j}$.

7 The Jacobi number, $\mathscr{E}$, has been defined only in the case where no $y_{j}$ is missing from each $F_{i}$. If a polynomial does not involve one of the indeterminates, we shall define its order in that letter to be -1 , in which case $\mathscr{J}$ would always have a meaning. In such a situation, $\mathscr{G}$ may be less than $\mathscr{J}$.

8 If one of $y_{1}, y_{2}$ is missing from one of $F_{1}, F_{2}$, and $\mathscr{J}$ is defined as in footnote 7 ), then $\mathscr{G}$ is a better bound than $\mathscr{J}$, since in this case $\mathscr{G} \leqq \mathscr{J}$. 
an event, $r_{1}-\alpha_{11}=0, r_{2}-\alpha_{12}=0, r_{1}-\alpha_{21}>0, r_{2}-\alpha_{22}>0$. Hence, $w=\min \left(r_{1}-\alpha_{21}, r_{2}-\alpha_{22}\right)$ and $\mathscr{G}=r_{1}+r_{2}-\min \left(r_{1}-\alpha_{21}, r_{2}-\alpha_{22}\right)=$ $\max \left(r_{1}+\alpha_{22}, r_{2}+\alpha_{21}\right)=\mathscr{J}$.

\section{REFERENCES}

1. R. M. Cohn, Manifolds of difference polynomials, Trans. Math. Soc. 64 (1948), 133-172.

2. J. F. Ritt, Differential algebra, Colloq. Publi. Amer. Math. Soc., vol. XXXIII.

3. J. F. Ritt, and J. L. Doob, Systems of algebraic difference equations, Amer. J. Math. 55 (1933), 505-514.

4. J. F. Ritt and H. W. Raudenbush, Ideal theory and algebraic difference equations, Trans, Amer. Math. Soc. 46 (1939), 445-452.

5. J. F. Ritt, Jacobi's problem on the order of a system of differential equations, Ann Math. 26 (1935), 305-312.

DREW UNIVERSITY 


\section{PACIFIC JOURNAL OF MATHEMATICS}

\section{EDITORS}

\section{David Gilbarg}

Stanford University

Stanford, California

\section{R. A. Beaumont}

University of Washington

Seattle 5 , Washington

\author{
A. L. Whiteman
}

University of Southern California Los Angeles 7, California

L. J. Paige

University of California

Los Angeles 24, California

\author{
E. F. BECKENBACH \\ C. E. BURGESS \\ E. HEWITT \\ A. HORN
}

\author{
V. GANAPATHY IYER \\ R. D. JAMES \\ M. S. KNEBELMAN \\ L. NACHBIN
}

ASSOCIATE EDITORS
I. NIVEN

T. G. OSTROM

H. L. ROYDEN

M. M. SCHIFFER
E. G. STRAUS

G. SZEKERES

F. WOLF

K. YOSIDA

\section{SUPPORTING INSTITUTIONS}

\author{
UNIVERSITY OF BRITISH COLUMBIA \\ CALIFORNIA INSTITUTE OF TECHNOLOGY \\ UNIVERSITY OF CALIFORNIA \\ MONTANA STATE UNIVERSITY \\ UNIVERSITY OF NEVADA \\ OREGON STATE COLLEGE \\ UNIVERSITY OF OREGON \\ OSAKA UNIVERSITY \\ UNIVERSITY OF SOUTHERN CALIFORNIA
}

\author{
STANFORD UNIVERSITY \\ UNIVERSITY OF TOKYO \\ UNIVERSITY OF UTAH \\ WASHINGTON STATE COLLEGE \\ UNIVERSITY OF WASHINGTON \\ * * * \\ AMERICAN MATHEMATICAL SOCIETY \\ CALIFORNIA RESEARCH CORPORATION \\ HUGHES AIRCRAFT COMPANY \\ SPACE TECHNOLOGY LABORATORIES
}

Mathematical papers intended for publication in the Pacific Journal of Mathematics should be typewritten (double spaced), and the author should keep a complete copy. Manuscripts may be sent to any one of the four editors. All other communications to the editors should be addressed to the managing editor, L. J. Paige at the University of California, Los Angeles 24, California.

50 reprints per author of each article are furnished free of charge; additional copies may be obtained at cost in multiples of 50 .

The Pacific Journal of Mathematics is published quarterly, in March, June, September, and December. The price per volume (4 numbers) is $\$ 12.00$; single issues, $\$ 3.50$. Back numbers are available. Special price to individual faculty members of supporting institutions and to individual members of the American Mathematical Society: $\$ 4.00$ per volume; single issues, $\$ 1.25$.

Subscriptions, orders for back numbers, and changes of address should be sent to Pacific Journal of Mathematics, 2120 Oxford Street, Berkeley 4, California.

Printed at Kokusai Bunken Insatsusha (International Academic Printing Co., Ltd.), No. 6, 2-chome, Fujimi-cho, Chiyoda-ku, Tokyo, Japan.

PUBLISHED BY PACIFIC JOURNAL OF MATHEMATICS, A NON-PROFIT CORPORATION

The Supporting Institutions listed above contribute to the cost of publication of this Journal, but they are not owners or publishers and have no responsibility for its content or policies. 


\section{Pacific Journal of Mathematics}

\section{Vol. 9, No. $2 \quad$ June, 1959}

Lee William Anderson, On the breadth and co-dimension of a topological lattice

Frank W. Anderson and Robert L. Blair, Characterizations of certain lattices

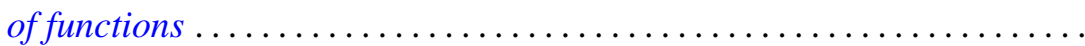

Donald Charles Benson, Extensions of a theorem of Loewner on integral

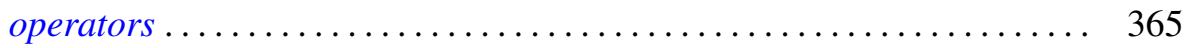

Errett Albert Bishop, A duality theorem for an arbitrary operator ........ 379

Robert McCallum Blumenthal and Ronald Kay Getoor, The asymptotic distribution of the eigenvalues for a class of Markov operators ........

Delmar L. Boyer and Elbert A. Walker, Almost locally pure Abelian

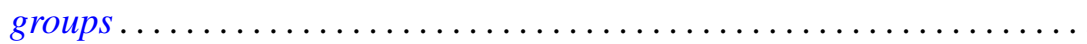

Paul Civin and Bertram Yood, Involutions on Banach algebras ........... Lincoln Kearney Durst, Exceptional real Lehmer sequences .... 415

Eldon Dyer and Allen Lowell Shields, Connectivity of topological

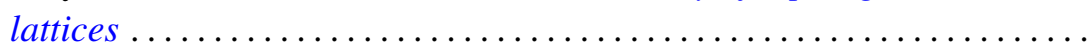

Ronald Kay Getoor, Markov operators and their associated

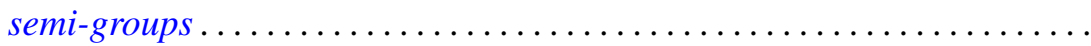

Bernard Greenspan, A bound for the orders of the components of a system of algebraic difference equations

Branko Grünbaum, On some covering and intersection properties in

Minkowski spaces ............................

Bruno Harris, Derivations of Jordan algebras ..............

Henry Berge Helson, Conjugate series in several variables.

Isidore Isaac Hirschman, Jr., A maximal problem in harmonic analysis.

II .

Alfred Horn and Robert Steinberg, Eigenvalues of the unitary part of a matrix

Edith Hirsch Luchins, On strictly semi-simple Banach algebras ...

William D. Munro, Some iterative methods for determining zeros of

functions of a complex variable...

John Rainwater, Spaces whose finest uniformity is metric .

William T. Reid, Variational aspects of generalized convex functions ....

A. Sade, Isomorphisme d'hypergroupoï des isotopes ...... . .

Isadore Manual Singer, The geometric interpretation of a special

connection . . .

Charles Andrew Swanson, Asymptotic perturbation series for characteristic

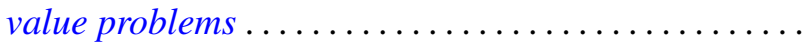

